\title{
Status and performance of the High Energy Particle Detector (HEPD) on-board the CSES-01 satellite
}

\author{
A. Sotgiü ${ }^{*}$, G. Ambrosi ${ }^{c}$, R. Battiston ${ }^{d, e}$, S. Bartocci ${ }^{a, k}$, L. Basara ${ }^{d}$, W.J. Burger ${ }^{d}$, D. \\ Campana $^{f}$, L. Carfora ${ }^{a, b}$, M. Casolino ${ }^{d}$, G. Castellini ${ }^{m}$, P. Cipollone $^{a}$, L. Conti $^{a, k}$, A. \\ Contin $^{h, i}$, C. De Donato ${ }^{a}$, F. De Persio ${ }^{a}$, C. De Santis ${ }^{a}$, B. Di Ruzza ${ }^{d, e}$, F.M. Follega ${ }^{d, e}$, \\ C. Guandalini ${ }^{h}$, M. Ionica ${ }^{c}$, R. luppa ${ }^{d, e}$, G. Laurenti $^{h}$, I. Lazzizzera ${ }^{d, e}$, M. Lolli ${ }^{h}$, C. \\ Manea $^{d}$, L. Marcelli ${ }^{a}$, M. Martucci ${ }^{a}$, G. Masciantonio ${ }^{a}$, M. Mergé ${ }^{a}$, G. Osteria ${ }^{f}$, L. \\ Pacini $^{m}$, F. Palma ${ }^{a, b}$, F. Palmonari ${ }^{h, i}$, B. Panico ${ }^{f}$, A. Parmentier ${ }^{a}$, L. Patrizii ${ }^{h}$, F. \\ Perfetto $^{f}$, P. Picozza ${ }^{a, b}$, M. Piersanti ${ }^{a}$, M. Pozzato ${ }^{h}$, M. Puel ${ }^{d}$, I. Rashevskaya ${ }^{d}$, E.

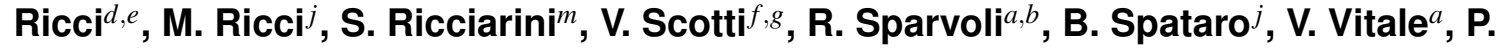 \\ Zuccon $^{d, e}, \mathbf{S}$. Zoffoli ${ }^{m}$ \\ ${ }^{a}$ INFN - Sezione di Roma Tor Vergata, V. della Ricerca Scientifica 1, 00133, Rome, Italy \\ ${ }^{b}$ Università di Roma Tor Vergata, V. della Ricerca Scientifica 1, 00133 Rome, Italy \\ ${ }^{c}$ INFN Sezione di Perugia, V. A. Pascoli, 06123, Perugia, Italy \\ ${ }^{d}$ INFN - TIFPA, V. Sommarive 14, 38123 Povo (Trento), Italy \\ ${ }^{e}$ Università di Trento, V. Sommarive 14, 38123 Povo (TN), Italy \\ ${ }^{f}$ INFN - Sezione di Napoli, V. Cintia, 80126, Naples, Italy \\ ${ }^{g}$ Università di Napoli Federico II, C.so Umberto I, 40, 80138, Naples, Italy \\ ${ }^{h}$ INFN - Sezione di Bologna, V.le Berti Pichat 6/2, Bologna, Italy \\ ${ }^{i}$ Università di Bologna, V.le Berti Pichat 6/2, Bologna, Italy \\ ${ }^{j}$ INFN - LNF, V. E. Fermi, 40, 00044 Frascati (RM), Italy \\ ${ }^{k}$ Uninettuno University, C.so V. Emanuele II, 39, 00186, Rome, Italy \\ ${ }^{l}$ IFAC-CNR, V. Madonna del Piano, 10, 50019 Sesto Fiorentino (FI), Italy \\ ${ }^{m}$ Italian Space Agency, V. del Politecnico, 00133 Rome, Italy \\ E-mail: alessandro.sotgiuaroma2.infn.it
}


CSES-01 (China Seismo-Electromagnetic Satellite) is a space mission dedicated to the study of the ionospheric environment, searching for disturbances that can be correlated to seismic activity. For this reason, the satellite is equipped with several instruments to monitor various physical parameters including the Earth's electric and magnetic field, ionospheric plasma principal parameters (i.e. density, temperature, and ions composition) and high energy particle flux fluctuations. The High Energy Particle Detector (HEPD), built by the Italian CSES-Limadou collaboration, is designed to detect mostly electrons in the energy range between $3 \mathrm{MeV}$ and $100 \mathrm{MeV}$ and protons in the energy range between $30 \mathrm{MeV}$ and $300 \mathrm{MeV}$. The detector is composed of a tracking system, a segmented layer of plastic scintillator (used for the trigger), a range calorimeter and a veto system.

After CSES-01 launch (February 2, 2018), the apparatus underwent the commissioning phase where its configuration was adjusted in order to guarantee optimal operational conditions in the space environment. Now the satellite is in stable data-taking mode.

In this work a description of the HEPD detector will be reported, focusing on the results of the electron and proton test beam calibrations. In addition, a particular relevance will be given to the presentation of the HEPD in-flight performance, such as its good capability in particle identification. The high statistic collected by HEPD, thanks to its large acceptance window, makes it perfectly suitable for the investigation of fluctuations in the charged particle fluxes.

36th International Cosmic Ray Conference -ICRC2019-

July 24th - August 1st, 2019

Madison, WI, U.S.A.

${ }^{*}$ Speaker. 


\section{The High-Energy Particle Detector}

The High-Energy particle detector[1] is the Italian contribution to the China Seismo-Electromagnetic Satellite (CSES) space mission. The mission is devoted to monitoring perturbation in the Earth's ionosphere environment, such as electromagnetic field and plasma density perturbations or trapped particle fluctuations, with the aim to study possible correlations with seismic events[2]. HEPD is one of the two particle detectors on-board the CSES satellite and is designed to measure mainly electrons and protons in the energy range 3-100 MeV and 30-300 MeV respectively. For this purpose, it is composed of the following set of sub-detectors as shown in Fig. 1:

- a tracker system composed of two layers of double-sided silicon micro-strip sensors, placed at the top of the instruments for the reconstruction of the particle incident direction

- a trigger plane composed of a segmented plastic scintillator layer, with each of the 6 segments (paddles) read-out by two photomultiplier tube (PMT) and independently from the others

- a range-calorimeter divided into two sections. The upper one is made of 16 plastic scintillator planes (Upper Calo) read out by two PMTs placed at the corners of each plane, the bottom section is made of a $3 \times 3$ matrix of LYSO inorganic crystals read out by one PMT placed at the bottom face

- a veto system, surrounding the whole calorimeter, composed of five planes of plastic scintillators (four lateral planes and a bottom one)
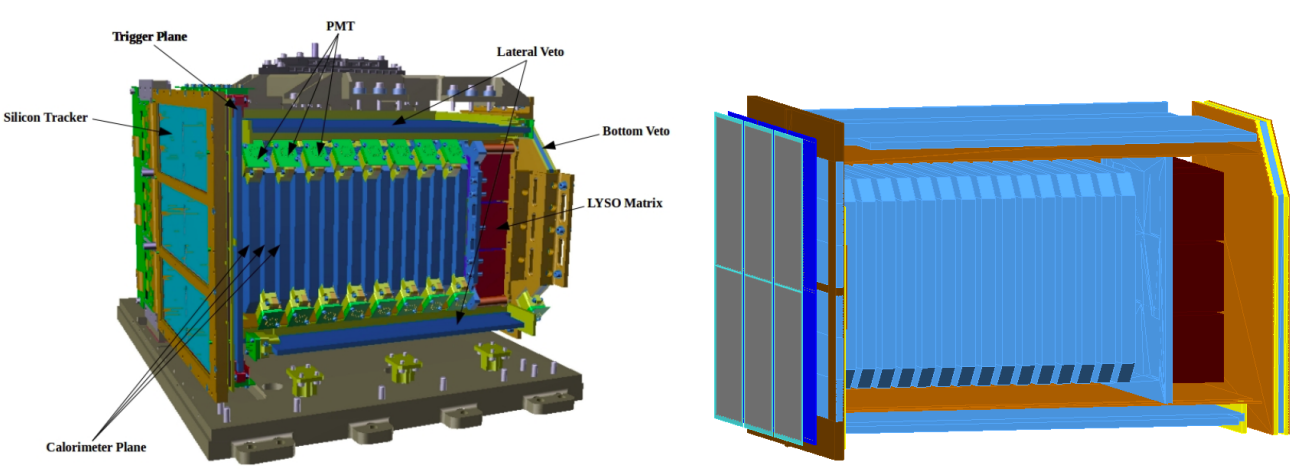

Figure 1: Left: CAD scheme of the HEPD detector including all the sensitive detectors and the mechanical structures. Right: The Monte Carlo geometry of HEPD, implemented by means of the Geant 4 software, that was used for the calibration of the detector. In both images, the frontal veto plane has been removed to allows the view of the internal detector structure.

\section{Detector calibration}

The energy calibration for the HEPD calorimeter included different steps. At first, for each PMT channel, the pedestal was measured and subtracted through an acquisition of events with an artificially generated trigger. Then, runs of atmospheric muons were acquired to equalize the PMTs 
response to a MIP particle (the same energy is expected to be released in each plane by a minimum ionizing particle). Finally, the detector underwent the test beam campaign and was irradiated with particles with well-known energies: electrons at the Beam Test Facility in Frascati (from 30 to 120 $\mathrm{MeV}$ ) and protons at the Proton Therapy Center in Trento (from 37 to $228 \mathrm{MeV}$ ).

A detailed Monte Carlo simulation of the detector during the test beam was implemented by means of the Geant 4 package. Both the detector and the incident beam configurations (i.e. beam energy, spot size, incident position on the HEPD window, etc.) were reproduced accurately, in order to tune the Monte Carlo with the test beam data. In particular, for all the scintillator planes of the Upper Calo, the propagation of the light inside each scintillator plane, as well as the light collected by each PMT, were implemented in the simulation, to obtain a calibration curve for the conversion of photo-electrons into ADC, for all the PMTs. This was done in the following way: for each energy of the test beam, the distribution of the signal in each PMT was fitted with a Gaussian function. The same was done with the distribution of photo-electrons obtained from the simulation. Then, the mean values obtained by the Gaussian fits were used to produce the calibration curve.

Fig. 2 shows as an example the calibration curve for a specific PMT of the Upper Calo.
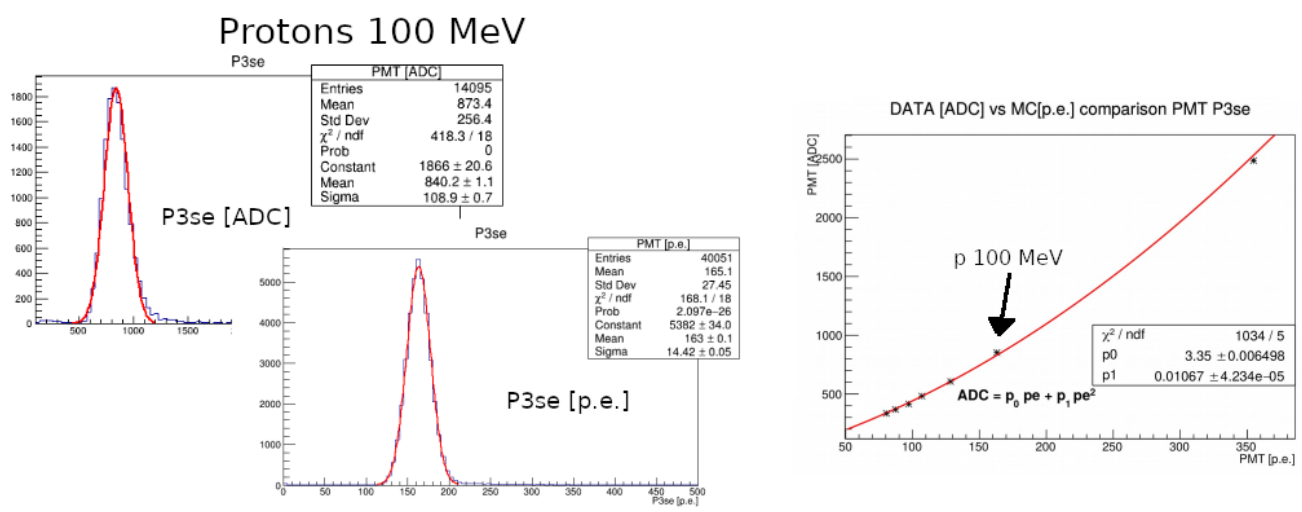

Figure 2: Left: distributions of ADC signal measured at the test beam and photo electrons collected in the Monte Carlo simulation for a specific PMT of the HEPD calorimeter with a $100 \mathrm{MeV}$ proton run; both distributions were fitted with a Gaussian. Right: the calibration curve for the conversion p.e. to ADC for the same PMT, obtained by using the mean value of the Gaussian fits for all the test beam energies.

Fig. 3 shows a very good agreement between acquired data (blue curves) and reconstructed ADC distributions (red curves) after the Monte Carlo digitization.

The Monte Carlo was then used for the energy calibration of the calorimeter. Fig. 4 shows the calibration curve of the Upper Calo of HEPD. The $\mathrm{X}$ axis reports the energy deposited inside the scintillator tower as estimated from the Monte Carlo simulation. The Y axis reports the sum of the signal deposited in the scintillator tower (i.e. the sum of all the PMT signals) as measured during the test beam.

The points representing muons and protons with energies of $174 \mathrm{MeV}, 202 \mathrm{MeV}$, and $228 \mathrm{MeV}$ are not contained in the Upper Calo. This is the reason why they released just a fraction of the total energy. The red points in the figure refer to data acquired with the HOT (main) side of the HEPD electronics powered on, while the blue points refer to data acquired with the COLD (spare) side of the electronics. The presence of the spare side is quite common for a space detector because 
redundancy is the best way to avoid permanent failures in the electronics subsystems. Fig. 4 also shows that the previous applied step of the PMTs equalitazion, cancels the different responses between the two sides of the electronics as the HOT and COLD points at the same energy are almost overlapped.
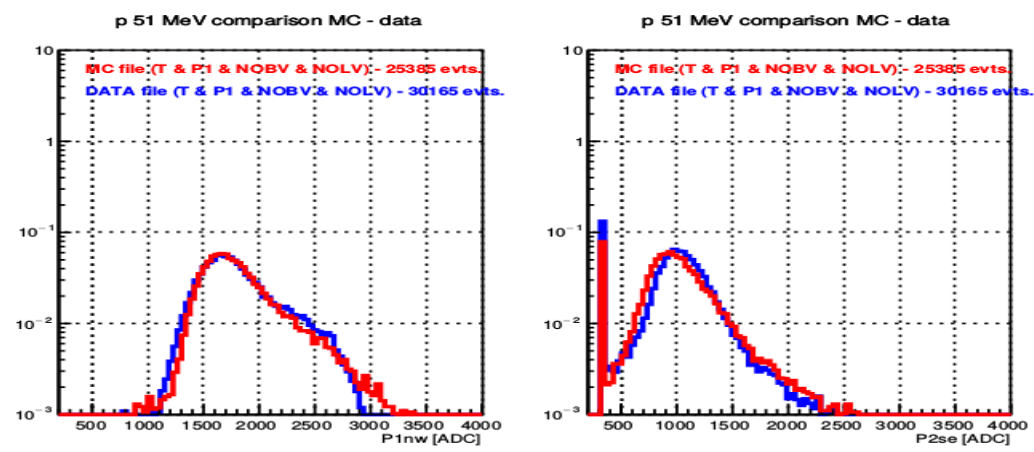

Figure 3: Comparison between distributions of acquired data (blue) and reconstructed ADC signal from $\mathrm{MC}$ (red) for two PMTs of the first two calorimeter planes, with a proton run of $51 \mathrm{MeV}$.

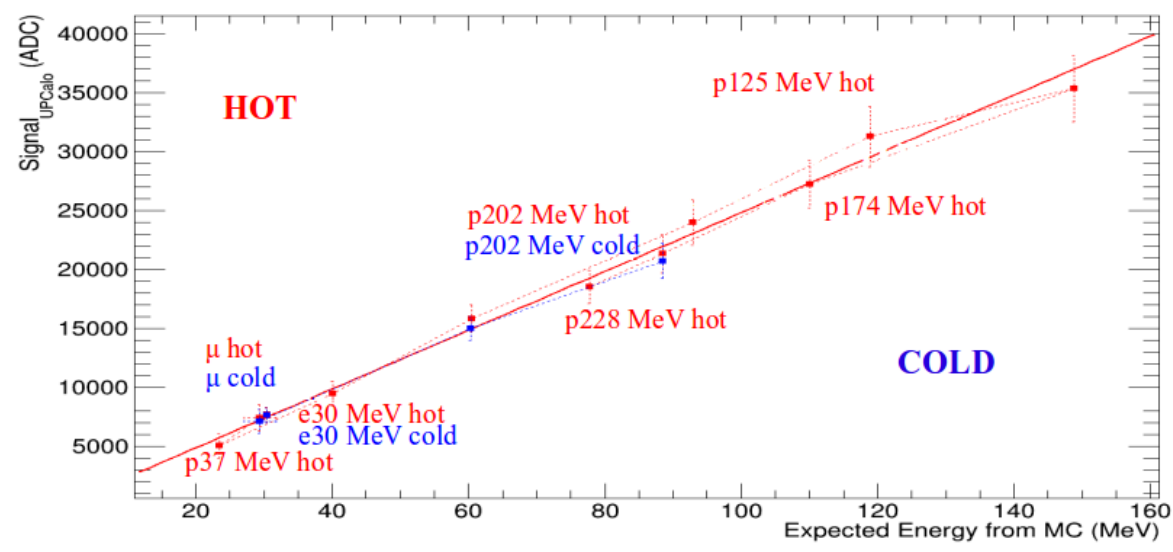

Figure 4: Calibration curve used for the energy reconstruction deposited in the scintillator tower of HEPD. Both the hot (red points) and cold (blue points) side of the HEPD electronics have been calibrated during the test beams.

\section{In-flight performances}

The CSES satellite was launched on February 2, 2018. Since it was in orbit, the HEPD detector response is continuously monitored. Calibration runs, where the pedestals and the associated RMS of each PMTs are determined, are repeated every orbit during the passage in an equatorial position $\left(-30^{\circ}<\right.$ latitude $\left.<30^{\circ}\right)$ where the acquisition rate is lower. Furthermore, the equalization is also checked by selecting a sample of in-flight minimum ionizing particle (high energy protons that do not interact in the instrument) and controlling that the MPV of the signal distribution is stable in time. Fig. 5 shows the Landau fit of the signal distribution of a MIP-like particle, measured in the two PMTs of the ninth plane. 

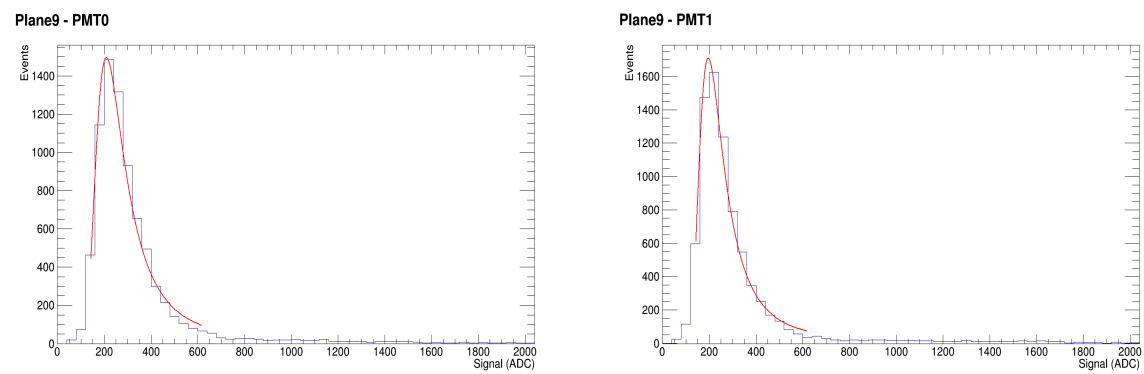

Figure 5: Distribution of the signal in two PMTs of the ninth plane, after selecting in-flight minimum ionizing particles. The MPV of the landau fit is used for checking the equalization.

Particle identification is not a problem for HEPD due to the different energy range of detectable electrons and protons. In fact, while the latter are slow in the HEPD energy range, electrons are relativistic and the release of energy plane by plane is quite constant.

Fig. 6 shows, for example, the coherence between the trapped proton geographical distribution inside the South Atlantic Anomaly, measured by HEPD (right) and obtained through the SPENVIS AP-8 model (left).

Fig. 7 reports the HEPD preliminary galactic proton flux measured for a single day of August 2018 compared to the HelMod model[3] calculated during the period of July-August 2018. The selection of galactic protons requires:

- protons fully contained in the calorimeter (no hit in any veto plane)

- no spurious hit on more than one trigger paddle or LYSO crystals (to remove secondaries and interacting events)

- particles coming from outside the magnetosphere (energy greater than 1.3 times the local geomagnetic cut-off rigidity)

It can be noted that, although a detailed study of the detector efficiency is required, the flux shows a good agreement with the HelMod model. In addition, the large acceptance of the HEPD detector results in high statistics, allowing a daily flux calculation.

\section{Conclusions}

This paper reports the procedures applied for the HEPD calibration, both for test beam ground calibration and the operations carried out in orbit. A very detailed MC simulation was performed in order to obtain a reliable energy calibration, which is also checked continuously with in-flight acquisitions of minimum ionizing particles.

Some in-flight results, for what concerns trapped protons distributions in the South Atlantic Anomaly and a preliminary galactic proton flux, have been compared with models. The analysis of the HEPD data acquired after the CSES launch and the consistency with theoretical models show that the detector is working as expected. The large acceptance window of HEPD provides a very high statistics, also on a daily base, allowing very precise measurement of transient effects and a good sensitivity to different particle populations. 
AP-8 MIN Model

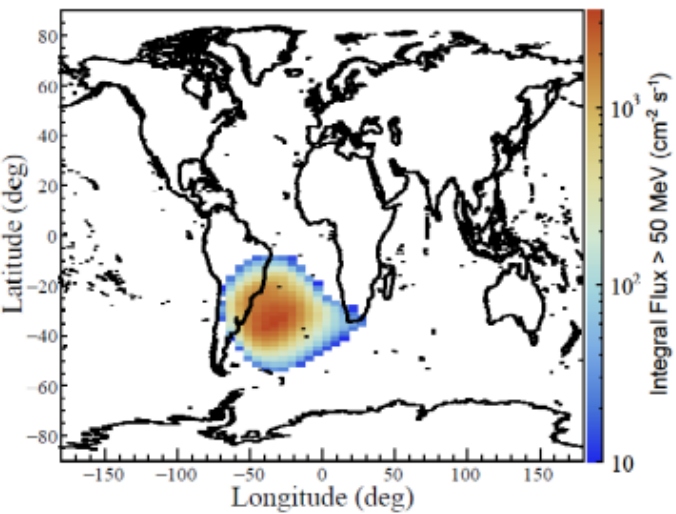

HEPD Data

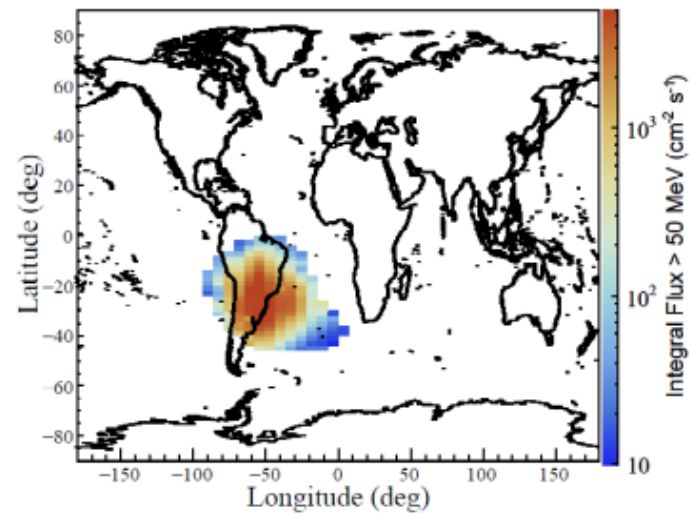

Figure 6: Comparison between trapped protons inside the SAA obtained through the AP-8 MIN model with data from 2015 (left) and HEPD data from August 2018 (right). The shift of the SAA region that can be observed between model and HEPD data is explained by the fact that the two plots are obtained in two different periods.

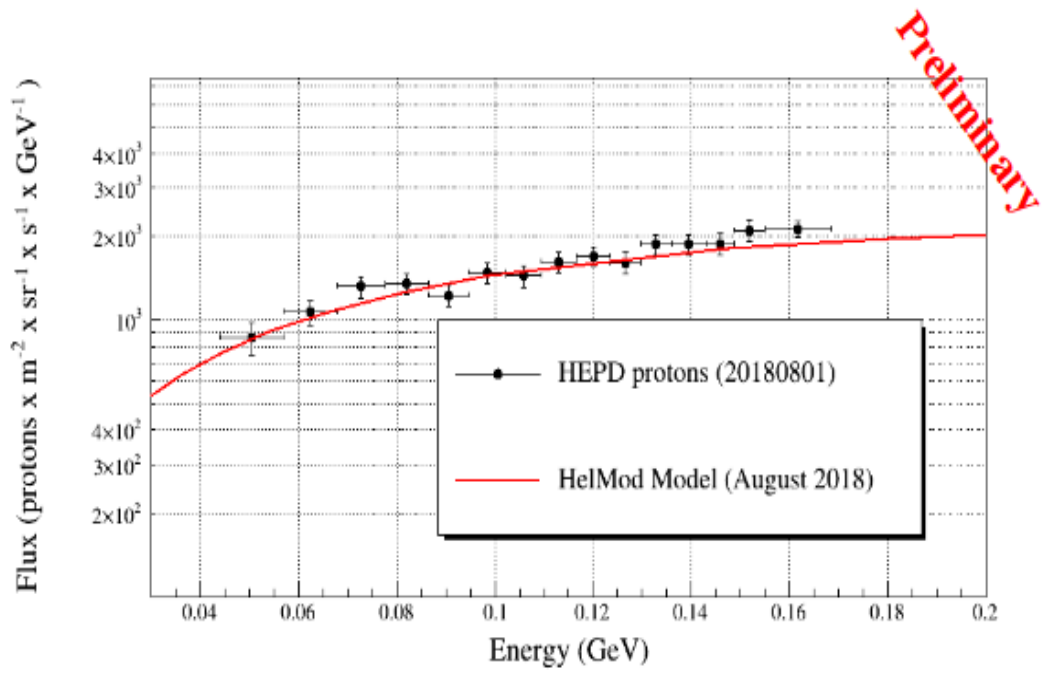

Figure 7: HEPD galactic proton flux (black points), obtained for the $1^{\text {st }}$ of August 2018, compared with the theoretical flux from the HelMod model (red solid line) calculated during the period July-August 2018.

\section{References}

[1] P. Picozza, R. Battiston et al., Scientific goals and in-orbit performance of the High-Energy Particle Detector on board the CSES satellite, The Astrophysical Journal Supplement Series - In Press

[2] X. Shen et al., Preliminary proposal of scientific data verification in CSES mission, Earthquake Science 28.

[3] M. J. Boschini, S. Della Torre, M. Gervasi, G. La Vacca and P. G. Rancoita, The HelMod Model in the Works for Inner and Outer Heliosphere: from AMS to Voyager Probes Observations Advances in Space Research - In Press arXiv:1903.07501 